University of Wollongong

Research Online

Australian Institute for Innovative Materials -

Papers

Australian Institute for Innovative Materials

$1-1-2014$

\title{
Mechanically strong high performance layered polypyrrole nano fibre/ graphene film for flexible solid state supercapacitor
}

Sha Li

University of Wollongong, sl739@uowmail.edu.au

Chen Zhao

University of Wollongong, cz995@uowmail.edu.au

Kewei Shu

University of Wollongong, ks323@uowmail.edu.au

Caiyun Wang

University of Wollongong, caiyun@uow.edu.au

Zaiping Guo

University of Wollongong, zguo@uow.edu.au

See next page for additional authors

Follow this and additional works at: https://ro.uow.edu.au/aiimpapers

Part of the Engineering Commons, and the Physical Sciences and Mathematics Commons

Research Online is the open access institutional repository for the University of Wollongong. For further information contact the UOW Library: research-pubs@uow.edu.au 


\title{
Mechanically strong high performance layered polypyrrole nano fibre/graphene film for flexible solid state supercapacitor
}

\author{
Abstract \\ Paper like flexible electrode becomes one of the most important research objects recently in request of \\ the fast expanding market of portable electronics. Flexible solid state supercapacitors are shortlisted as \\ one of the most promising energy devices to power electronics with medium to high power density \\ requirements. In this work, we developed a simple but effective way to produce a mechanically strong and \\ electrochemically active RGO/polypyrrole (PPy) fibre paper. A well-bedded microstructure was created \\ with interlaced polypyrrole fibres evenly distributed between the graphene layers. Such microstructure can \\ create enormous amount of pores and therefore provides larger interfaces for charge carrier storage/ \\ release. The effects of polypyrrole fibres on the film's morphologies, mechanical properties and \\ electrochemical performance were discussed. A solid state supercapacitor was demonstrated using such \\ paper electrodes and a gel type electrolyte - phosphate acid (H3PO4) infused polyvinyl alcohol (PVA). It \\ showed a high capacitance ( $345 \mathrm{~F} \mathrm{~g}-1$ ) and an excellent cycling stability ( $9.4 \%$ drop after 1000 cycles).

\section{Keywords} \\ performance, high, strong, mechanically, polypyrrole, nano, fibre, layered, graphene, supercapacitor, film, \\ flexible, solid, state \\ Disciplines \\ Engineering | Physical Sciences and Mathematics

\section{Publication Details} \\ Li, S., Zhao, C., Shu, K., Wang, C., Guo, Z., Wallace, G. G. \& Liu, H. (2014). Mechanically strong high \\ performance layered polypyrrole nano fibre/graphene film for flexible solid state supercapacitor. Carbon, \\ 79 554-562.
}

\section{Authors}

Sha Li, Chen Zhao, Kewei Shu, Caiyun Wang, Zaiping Guo, Gordon G. Wallace, and Hua-Kun Liu 


\title{
Mechanically strong high performance layered polypyrrole nano fibre/graphene film for flexible solid state supercapacitor
}

\author{
Sha $\mathrm{Li}^{1,2}$, Chen $\mathrm{Zhao}^{2}$, Kewei $\mathrm{Shu}^{2}$, Caiyun Wang ${ }^{2 *}$, ZaiPing Guo ${ }^{1}$, Gordon.G.Wallace ${ }^{2}$, \\ HuaKun $\mathrm{Liu}^{1,2 *}$ \\ 1, Institute for Superconducting and Electronic Materials, University of Wollongong, \\ 2, ARC Centre of Excellence for Electromaterials Science, University of Wollongong
}

Corresponding Author: Prof. Hua Kun Liu

Phone: +61242214547

Fax: +61242215731

E-mail: hua_liu@uow.edu.au

Dr Caiyun Wang

Phone +61242981426

E-mail: caiyun@uow.edu.au 


\section{Abstract}

Paper like flexible electrode becomes one of the most important research objects recently in request of the fast expanding market of portable electronics. Flexible solid state supercapacitors are shortlisted as one of the most promising energy devices to power electronics with medium to high power density requirements. In this work, we developed a simple but effective way to produce a mechanically strong and electrochemically active RGO/polypyrrole (PPy) fibre paper. A well-bedded microstructure was created with interlaced polypyrrole fibres evenly distributed between the graphene layers. Such microstructure can create enormous amount of pores and therefore provides larger interfaces for charge carrier storage/release. The effects of polypyrrole fibres on the film's morphologies, mechanical properties and electrochemical performance were discussed. A solid state supercapacitor was demonstrated using such paper electrodes and a gel type electrolyte - phosphate acid $\left(\mathrm{H}_{3} \mathrm{PO}_{4}\right)$ infused polyvinyl alcohol (PVA). It showed a high capacitance $\left(345 \mathrm{~F} \mathrm{~g}^{-1}\right)$ and an excellent cycling stability (9.4\% drop after 1000 cycles).

\section{Introduction}

Over the last decade, low cost, efficient and miniature energy storage devices become the key research direction worldwide, which is motivated by the fast expanding market of portable electronics. Flexible electronics such as roll-up display, electronic paper, stretchable integrated circuits, and wearable systems for personal multimedia or medical devices all need reliable energy supplies with good flexibility ${ }^{1-5}$. Supercapacitors generally possess moderate energy density ranging between 0.1 to $10 \mathrm{Wh} \mathrm{Kg}^{-16}$ but with high-energy-storage efficiencies and excellent cycling stabilities ${ }^{7}$. It is envisageable that flexible solid state supercapacitors with proper power outputs could be a promising power source for those flexible devices. 
Ever since the discovery of graphene, many research works have been carried out to develop supercapacitors using graphene or graphene based composites materials ${ }^{8}$. Graphene has the interesting one atom thick single layer hexagonal lattice structure with excellent physical and chemical properties, which makes it a versatile material for many applications ${ }^{9}$. As for supercapacitors, graphene are utilized as the electrode materials because of its fast charge carrier transportation, chemical stability and high specific surface areas ${ }^{8,10,11}$. The specific surface area of the exfoliated graphene nano sheets is superior to most of the carbon materials including carbon nanotubes, which is up to $2620 \mathrm{~m}^{2} \mathrm{~g}^{-1}$ theoretically ${ }^{9}$. The large surface areas provides enormous interfaces for charge carrier desorption/absorption and contribute largely to the charge capacitance.

Typically the average specific capacitance of supercapacitors reported with graphene based electrodes in both organic and aqueous electrolyte are all above $200 \mathrm{~F} \mathrm{~g}^{-18}$. Meanwhile, there have been remarkable breakthroughs in improving the energy densities of the graphene based supercapacitors as well. C. Liu et al reported a high quality graphene based capacitor which exhibited a specific energy density of $85.6 \mathrm{Wh} \mathrm{kg}^{-1}$ at room temperature ${ }^{12}$. Besides, graphene nano sheets are mechanically strong and possess high aspect ratio, which can be easily fabricated into freestanding papers via various methods. Those graphene papers are highly suitable for flexible solid state supercapacitors ${ }^{11,13}$.

However, the restacking of graphene nano sheets (GNs) is a critical problem for those highly compact flexible graphene papers, which limits its electrochemical performance. Nano-structure architecting of these graphene based flexible papers is one the important methods to solve this problem. There are two common approaches applied including integrating curly or porous GNs into papers or sandwiching fibre like nano material or nano particles between $\mathrm{GNs}^{14-21}$.

In this work, we choose the second pathway to avoid the possible damage to the mechanical properties. A layered structure, formed with polypyrrole (PPy) nano fibres and graphene nano sheets, was produced by a simple but effective method. Polypyrrole fibre is selected as the spacing material between GNs due to its low cost, ease of synthesis and high electric conductivity ${ }^{22-24}$. Also its pseudo- 
capacitive behaviour from the reversible redox reactions is an addition to achieve high capacitance ${ }^{23}$. The polypyrrole fibres with uniform thickness were synthesised using the surfactant micelles as shaping templates and mixed with negatively charged GNs dispersion for preparing flexible films. A gel type electrolyte, phosphate acid $\left(\mathrm{H}_{3} \mathrm{PO}_{4}\right)$ infused polyvinyl alcohol (PVA) is applied to make integrated flexible capacitors. Such capacitors demonstrate a high galvanostatic discharge specific capacitance of $345 \mathrm{~F} \mathrm{~g}^{-1}$. This value is higher than most of the supercapacitors composed of PPy/RGO electrodes reported to date ${ }^{25-32}$.

\section{Experimental}

\subsection{Preparation of PPy nano fibres and Graphene oxide (G0) dispersion}

Chemicals used for synthesizing polypyrrole nano fibres including pyrrole monomer, ammonium persulfate (APS) and cetrimonium bromide (CTAB) were all purchased from Sigma Aldrich. Pyrrole was distilled before polymerization to remove the impurities. $0.109 \mathrm{~g}(0.3 \mathrm{mmol}) \mathrm{CTAB}$ was added into $200 \mathrm{ml}$ deoxygenated water to form spherical micelles for wrapping pyrrole monomers, followed by the addition of $0.27 \mathrm{ml}(20 \mathrm{mM})$ pyrrole. The pyrrole/CTAB suspension was stirred and placed in an ice-water bath $\left(0^{\circ} \mathrm{C}\right) .0 .91 \mathrm{~g}(20 \mathrm{mM})$ APS was dissolved in $10 \mathrm{ml}$ water and added dropwisely into the pyrrole/CTAB suspension, and kept stirring the mixture for 24 hours for fully polymerization. The polymerization production was then washed by deionized water and ethanol to remove the surfactant residues and vacuum dried at $60^{\circ} \mathrm{C}$ for 12 hours.

Graphene oxide was synthesized from natural graphite powder by a modified Hummers method and referred to the exfoliation process developed by $\mathrm{Xu}$ et $a l^{33}$. The concentration of the as-prepared graphene oxide (GO) solution was quantified as $9.6 \mathrm{mg} \mathrm{ml}^{-1}$. The resulting homogeneous yellowbrown dispersion was stable for several months and ready for future processing. 


\subsection{Preparation and reduction of GO and PPy fibre/GO papers}

Both the thin PPy fibre/GO paper and GO paper were prepared by vacuum filtration method using polycarbonate filter membranes. $2 \mathrm{ml} \mathrm{GO}$ dispersion was diluted by $20 \mathrm{ml}$ water and vacuum filtered to produce a thin and smooth GO film. To make PPy fibre/GO paper, $10 \mathrm{mg}$ PPy fibre was added into $20 \mathrm{ml}$ water and ultrasonicated for 30 seconds. $2 \mathrm{ml} \mathrm{GO}$ was mixed with the PPy fibre dispersion and filtered $(\mathrm{PPy}: \mathrm{GO}=0.5: 1)$. The as prepared films were vacuum dried at $60^{\circ} \mathrm{C}$ for 12 hours. The GO and PPy fibre/GO films were reduced by being immersed in Hydriodic acid (HI, 47\%) at $4{ }^{\circ} \mathrm{C}$ for 20 minutes. The films were washed by water and ethanol to remove the acid residues. PPy/RGO films with different GO:PPy mass ratios (0.25:1, 0.5:1 and 0.75:1) were prepared using the same method. The GO:PPy was selected and investigated in this work based on its electrochemical performance and mechanical properties. The detailed discussion was provided in the supporting information.

\subsection{Construction of capacitor cells}

The procedures to fabricate the $\mathrm{PVA} / \mathrm{H}_{3} \mathrm{PO}_{4}$ gel and the supercapacitor device are similar to what we previously reported ${ }^{34}$. Briefly, $10 \mathrm{~g}$ PVA was dissolved into $100 \mathrm{ml}$ water at $90^{\circ} \mathrm{C}$. The PVA: $\mathrm{H}_{3} \mathrm{PO}_{4}$ ratio was 9:1. To construct the cell, all the paper electrodes were all immersed in the electrolyte overnight and dried afterwards. The gel electrolyte was spread on top of the dried films uniformly. Two electrodes with the semi-dried electrolyte layer were pressed to get a good attachment. Two pieces of stainless steel mesh wires were jointed to the back of the paper electrodes by highly conductive silver paste (Electron Microscope Sciences.co, USA). 


\subsection{Characterization and Cell testing}

Topographic heights of graphene oxide nano sheets were measured by the atom force microscope (Asylum Research, MFP-3D). Bright field and high resolution transmission electron microscope (TEM) images were taken by a JOEL JEM-2011 microscope with $120 \mathrm{KV}$ accelerating voltage. Morphologies of the as-prepared PPy fibre and PPy fibre/RGO films were observed with a field emission scanning electron microscope (FESEM, JOEL 7500). The Raman measurements were performed with a JOBIN YVON HR800 Confocal Raman system with $632.8 \mathrm{~nm}$ diode laser excitation on a 300 lines/mm grating at room temperature. X-ray photoelectron spectroscopy (XPS) was performed using a XPS system equipped with a hemispherical energy PHOIBOS100/150 analyser. The strain-stress curves of pure GO and PPy fibre/GO films were tested using a shimadzu EZ mechanical tester. Electrochemical testing including impedance spectroscopy (EIS), cyclic voltammetry $(\mathrm{CV})$ and cell charge/discharge performances were carried out by a $\mathrm{CH}$ Instrument $(\mathrm{CHI}$ 660B). 


\section{Results and discussions}

\subsection{Morphologies}
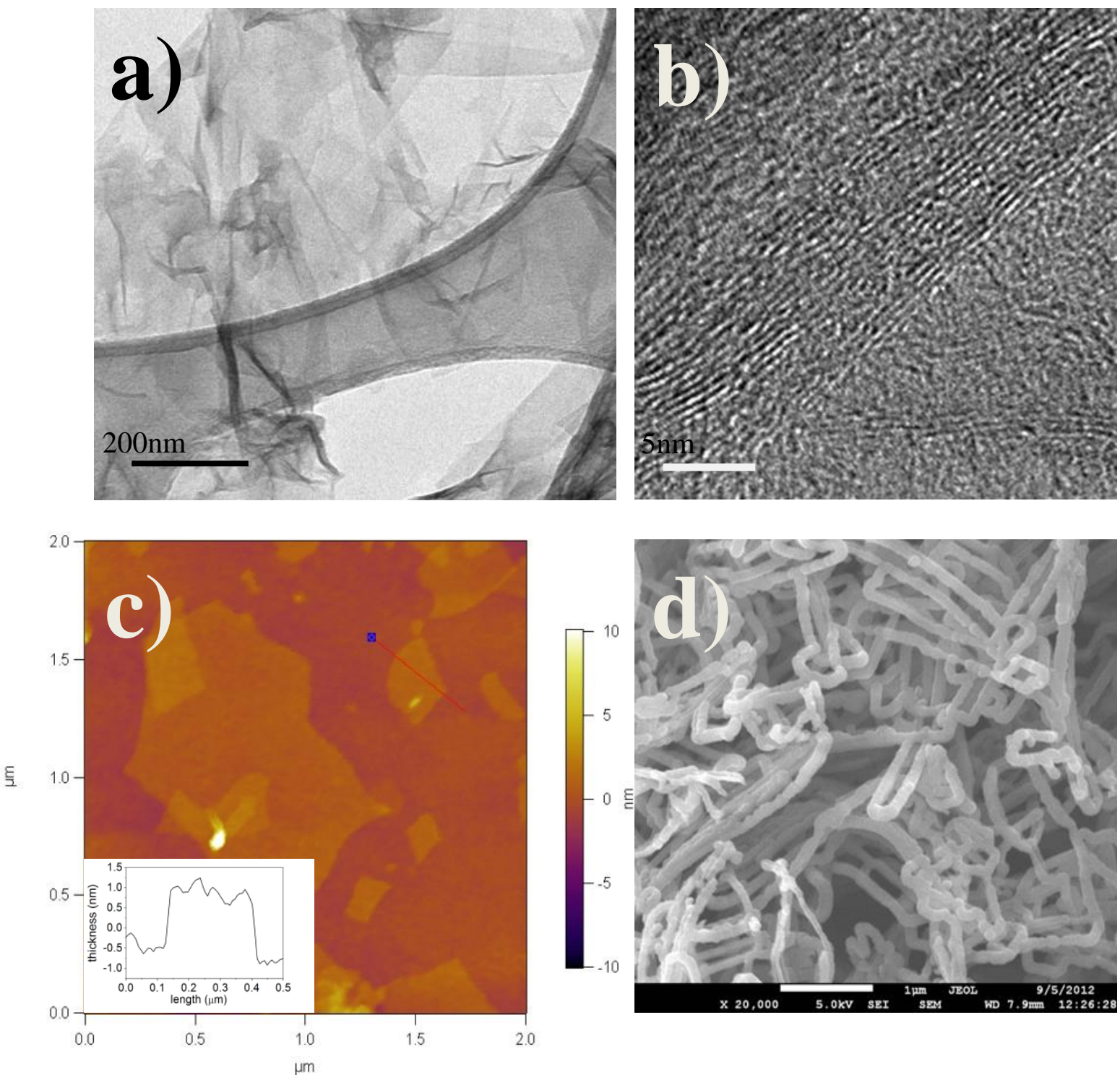

Figure .1 (a) Bright field transmission electron microscope image of exfoliated graphene oxide sheets. (b)

High resolution TEM image of exfoliated graphene oxide sheets along the profiles (c) AMF image of exfoliated graphene oxide layers (d) FESEM image of chemically synthesised polypyrrole nano fibres

Topological height and surface dimensions of the as-prepared graphene oxide sheets were measured using AFM and TEM respectively. Large sized 2D graphene oxide nano sheets are observed from bright field TEM image (Fig.1a). The graphene sheets are of sheer veil like morphology which ripples slightly producing wrinkles at the overlapped areas. Fig.1b showed the lateral high resolution TEM 
images of the dried graphene nano sheets, and the inter plane distance is measured to be $0.65 \mathrm{~nm}$. The thickness of graphene oxide sheets (in dispersion) is measured as $1.5 \mathrm{~nm}$ by AFM (Fig.1c). Such thickness can be assigned to a single layer hydrated graphene oxide sheets, representing the complete exfoliation of graphite oxide ${ }^{35}$. Due to the presence of CTAB, the pyrrole monomers can be attached onto the hydrophobic cores of surfactant micelles where the polymerization occurred. The produced polypyrrole takes the micelles nanofibre structure ${ }^{36}$. These nanofibres are uniform in diameters with an average thickness of approximately $100 \mathrm{~nm}$ (Fig.1d). The high aspect ratio of these fibres makes them easily to be interwoven together producing a porous network structure (Fig.1d).

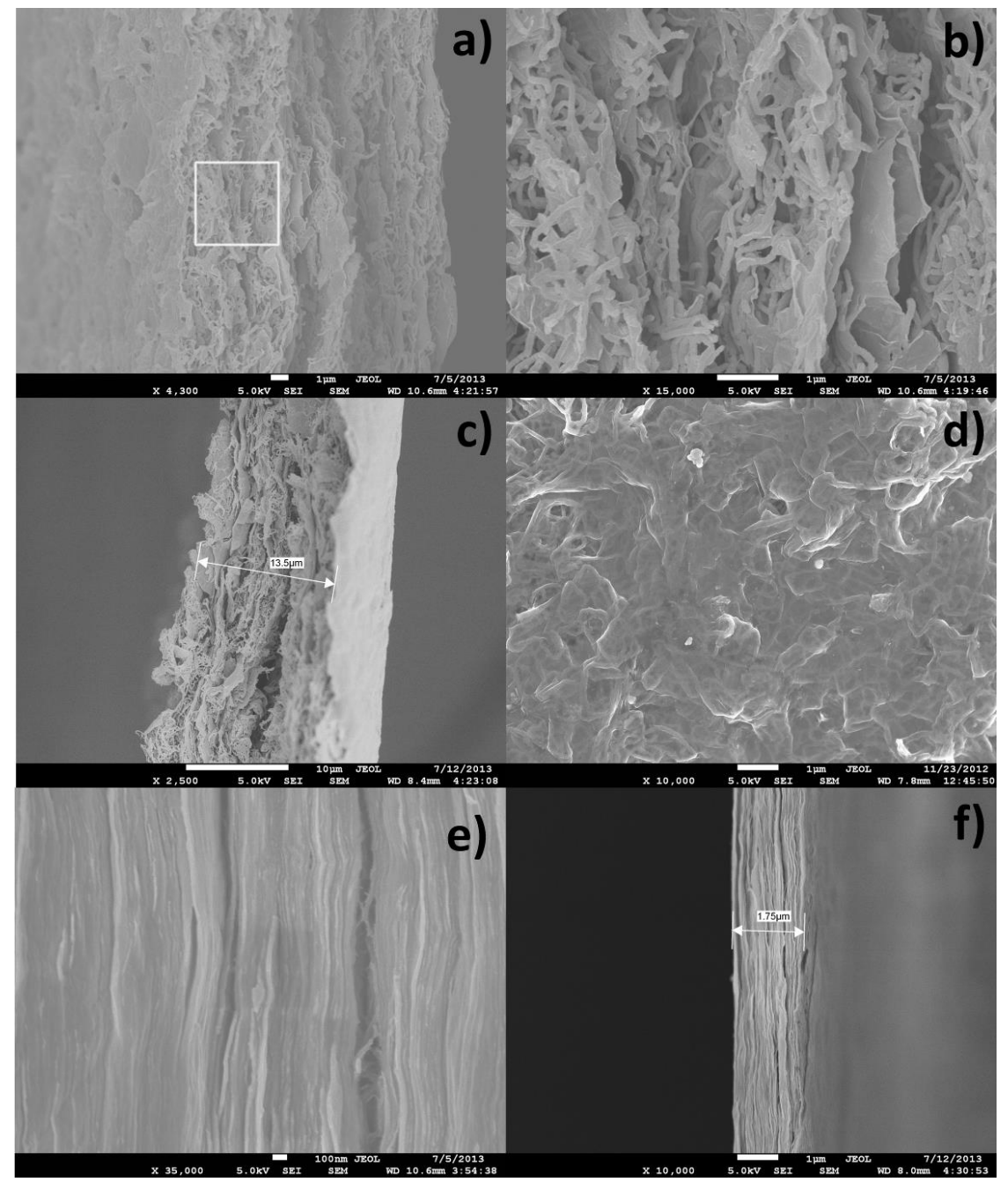

Figure .2 FESEM images showed (a) cross section of PPy fibre/RGO film (b) localized cross section of the area which defined by the pane in (a). (c) thickness of PPy fibre/RGO film. (d) surface of PPy fibre/RGO film. (e) cross section of pure GR film (f) thickness of pure RGO film. (RGO= reduced graphene oxide) 
Fig.2 showed the morphologies of pure RGO film and RGO/ PPy fibre films from different views. Fig.2 $\mathrm{a}$ and $\mathrm{b}$ are the cross-sectional images of the PPy fibre/RGO film. The interspaces between graphene layers are created by the evenly distributed PPy fibres, which significantly reduce the restacking of RGO layers comparing to the pure RGO films as showed in Fig.2e. After adding $50 \mathrm{wt} \%$ PPy fibre, the film thickness is enlarged by 7 times (Fig. $2 \mathrm{c}$ and f). The surface morphology of the RGO/PPy fibre film is showed in Fig.2d. The polypyrrole fibres are wrapped by the RGO sheets producing enlarged surface areas with large amount of concaves and convexities. Generally, the GO film assembled by vacuum filtration can induce restacking of graphene oxide sheets. The polypyrrole fibres have the function of spacing the RGO layers, creating a loose layered structure with pores and higher surface area. The specific surface area of both PPy/GO and pure GO film were measured as $157 \mathrm{~m}^{2} \mathrm{~g}^{-1}$ and $32 \mathrm{~m}^{2} \mathrm{~g}^{-1}$ via the methylene blue adsorption method ${ }^{37}$. The incorporation of PPy nanofibressignificantly increased the surface area, which clearly verified that PPy nano fibres effectively eased the restacking of graphene nano sheets during the film assembly.

\subsection{Mechanical property testing}
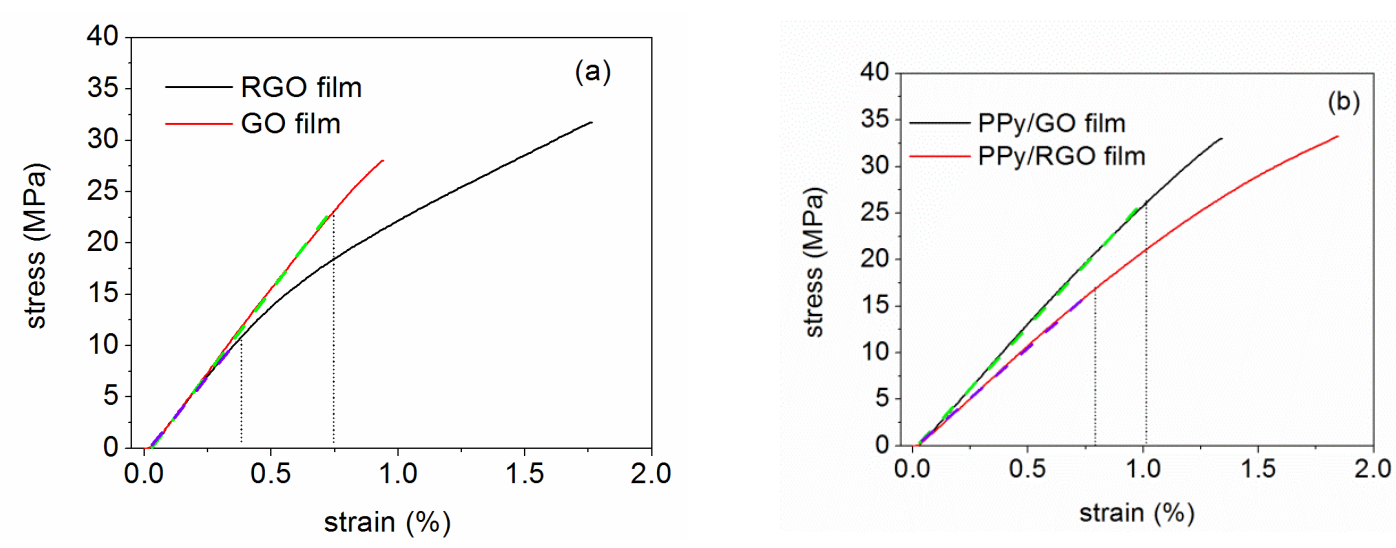

Figure.3 Strain stress curves of GO film (a) and PPy/GO film before and after reduction by HI.

According to the cross-sectional image of RGO films (Fig.3 a,b), it still retained the well-bedded structures after the reduction. The strain stress curves of RGO film and PPy/RGO film (Fig.3) both composed of a typical "washboard" pattern with the linear elastic regions and the self-reinforcing region $^{38}$. The young's modulus did not have significant decrease upon reduction, which are only $15 \%$ 
decrease for GO film, and 19\% for PPy/GO film, indicating that the elasticity of both films are retained well after being reduced by HI. HI is a residue free reducing agent capable of removing the carbonyl and carboxyl groups fast and efficiently without gas emission ${ }^{39}$. Thus, both the structures and mechanical properties of the films can be retained after being reduced by HI solution. The fracture strength and maximum elongation of pure GO film increased notably upon reduction, which possess the amplifications of $90 \%$ and $18 \%$ respectively as illustrated in table 1 . Such phenomenon indicates that the self-reinforce of the RGO film is superior to the GO film, which becomes tougher against fracture. Meanwhile, these two values of the PPy/RGO film are significantly larger than the pure RGO film, implying that the inter-layer distributed PPy nano fibres have the function of enhancing the toughness of the film.

Table 1 Conductivities and Mechanical properties of different type of flexible films

\begin{tabular}{|lllll|}
$\begin{array}{c}\text { Types of } \\
\text { films }\end{array}$ & $\begin{array}{c}\text { Conductivity } \\
\left(\mathrm{S} \mathrm{cm}^{-1}\right)\end{array}$ & $\begin{array}{l}\text { Young's } \\
\text { modulus } \\
(\mathrm{MPa})\end{array}$ & $\begin{array}{l}\text { Fracture } \\
\text { strength } \\
(\mathrm{MPa})\end{array}$ & $\begin{array}{l}\text { Maximum } \\
\text { Elongation } \\
(\%)\end{array}$ \\
\hline PPy/GO & $3.80 \times 10^{-2}$ & 2.6 & 34.9 & 1.34 \\
\hline PPy/RGO & 142.08 & 2.1 & 35.0 & 1.84 \\
\hline Pure GO & $1.11 \times 10^{-2}$ & 3.2 & 27.6 & 0.93 \\
\hline Pure RGO & 164.92 & 2.7 & 32.5 & 1.76 \\
\hline
\end{tabular}

The conductivity of both PPy/GO and pure GO film increases significantly after reduction as shown in Table.1. It is believed that the increased conductivity is induced by the removal of oxygen functionalities, as a result, the carbon lattice interlayer distance is reduced and the interaction among the $2 \mathrm{D}$ carbon planes is intensified ${ }^{40,41}$. The increase in conductivity is correlated to the decrease of $\mathrm{C} / \mathrm{O}$ ratio $^{42}$. Therefore, it can be concluded that the significant increase in conductivity of both the films reflects the high reduction efficiency of GO using $\mathrm{HI}$. 


\subsection{Chemical characterization}
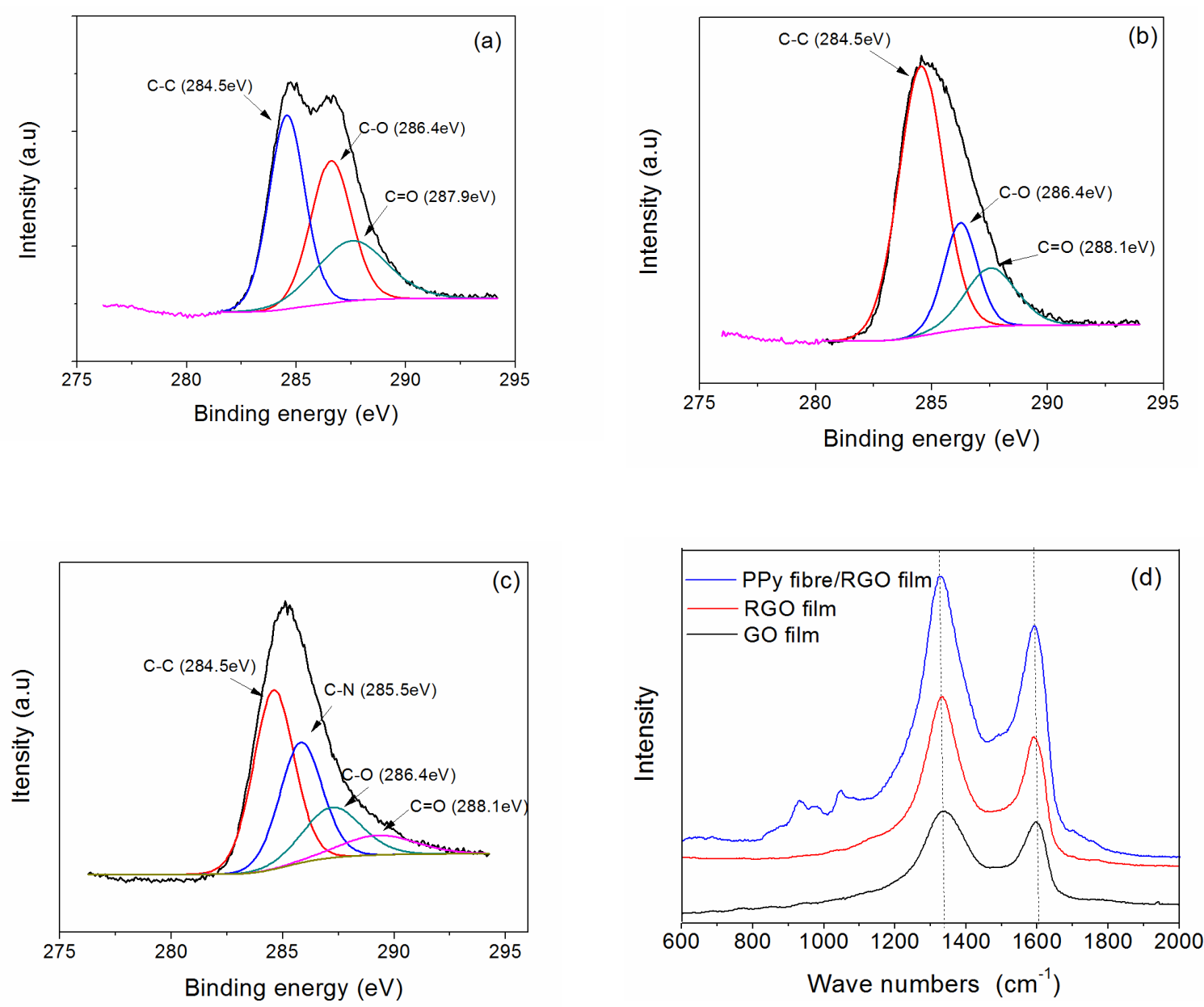

Figure .4 De-convoluted XPS C1s spectra of (a) Pure GO film (b) HI reduced RGO film (c) HI reduced PPy fibre/RGO film and (d) Raman Spectra of all these three types of films distinguished by different colours (blue for PPy fibre/RGO film, red for RGO film and black for GO film)

To further investigate the reduction effect of $\mathrm{HI}$ on these films, X-ray photoelectron spectroscopy was performed and analysed (Fig.4 a-c). Curve fittings of the spectrums were performed using the Gaussian-Lorantzian shape peaks based on the Shirley background correction. The peak representing the C-C bonding at $284.5 \mathrm{ev}$ is dominating in both the spectra of RGO film and PPy fibre/RGO film ${ }^{43}$, 44. The spectrum of PPy fibre/RGO film contains the characteristic peak of C-N backbone bonding with the binding energy of $285.5 \mathrm{ev}^{45}$. 
Pure GO film has two strong peaks that belong to the oxygen functionalities including C-O bonding at $286.4 \mathrm{ev}$ and $\mathrm{C}=\mathrm{O}$ bonding at $287.9 \mathrm{ev}$. These two peaks are significantly weakened after the reduction (Fig. 4 b, c) ${ }^{43}$. The $\mathrm{C} / \mathrm{O}$ ratio of pure $\mathrm{GO}$ film is 2.76 and rises to 8.76 after the reduction, reflecting the successful removal of oxygen functional groups ${ }^{39}$. It proves that the removal of oxygen functionalities using $\mathrm{HI}$ is efficient.

The structural information is also characterized by Raman spectrum (Fig.4d). The Raman spectrum of PPy fibre/RGO film displayed distinct peaks related to polypyrrole. The peak at $940 \mathrm{~cm}^{-1}$ demonstrates the $\mathrm{C}-\mathrm{H}$ out of plane deformation, while the peak at $998 \mathrm{~cm}^{-1}$ can be assigned to the pyrrole ring deformation. The peak at $1045 \mathrm{~cm}^{-1}$ corresponds to the $\mathrm{C}-\mathrm{H}$ in plane deformation, which is accompanied by a small shoulder peak at $1049 \mathrm{~cm}^{-1}$ related to the anion dopant. Polypyrrole has two strong peaks at $1314 \mathrm{~cm}^{-1}$ and $1600 \mathrm{~cm}^{-1}$ belonging to the ring stretching and C-C backbone stretching, respectively ${ }^{46}$. Those two peaks are overlapped with the D-band at $1340 \mathrm{~cm}^{-1}$ and G-band at $1580 \mathrm{~cm}^{-1}$ of graphene, suggesting the co-existence of PPy and graphene nano sheets ${ }^{47}$. The $\mathrm{G}$ band of 2D carbon materials corresponds to the first order scattering of the E2g mode of hexagonal carbon lattice and the in-plane bond stretching motion of the carbon $\mathrm{Sp} 2$ atoms, whilst the $\mathrm{D}$ peak is mainly attributed to the structural imperfections on the carbon basal plane ${ }^{47,48}$. After reduction, the intensity ratio between $D$ band and $G$ band $\left(I_{D} / I_{G}\right)$ is significantly increased for the pure GO film. This is mainly due to the decrease in the average size of the sp2 domains upon reduction of the exfoliated GO, which produces more imperfections because of the emerging of enormous edges and wrinkles from the fragmentized carbon sp2 domains. 


\subsection{Electrochemical properties}
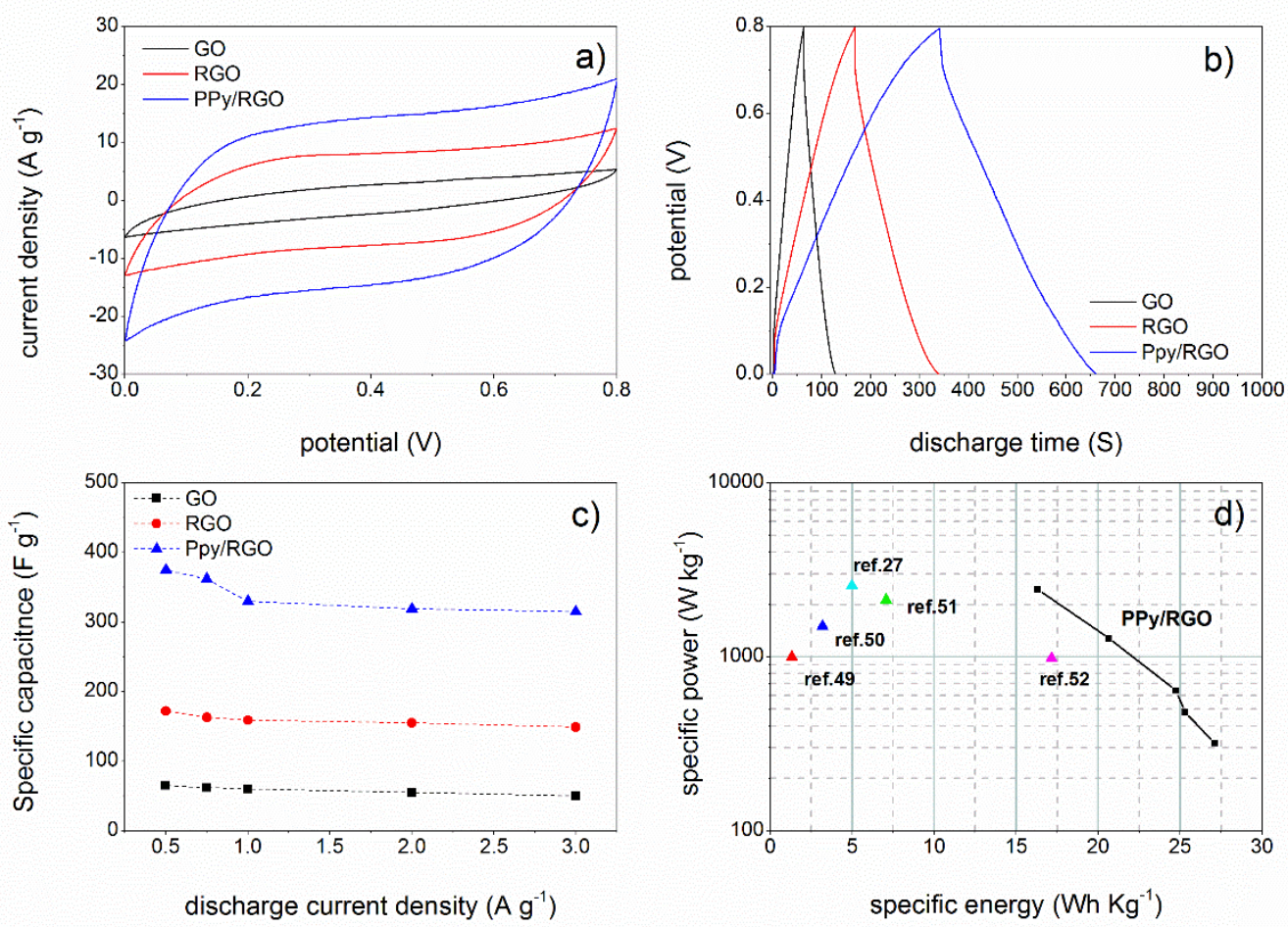

Figure.5 (a) Cyclic voltammogram curves of capacitors using $\mathrm{H}_{3} \mathrm{PO}_{4} \mathrm{PVA}$ gel as electrolyte with different flexible electrodes at the scan rate of $50 \mathrm{mV} \mathrm{S}^{-1}$, (b) Galvanic discharge curves at $1 \mathrm{~A} \mathrm{~g}^{-1}$ (c) Capacitances versus charge/discharge current densities and (d) Ragone plot of capacitor composed of PPy/RGO film electrode with the comparison to analogical solid-state supercapacitors) $)^{27,49-52}$

Fig.5a shows the CV curves of the flexible capacitors constructed with different types of film electrodes and $\mathrm{PVA} / \mathrm{H}_{3} \mathrm{PO}_{4}$ gel electrolyte at the scan rate of $50 \mathrm{mV} \mathrm{S}{ }^{-1}$. The capacitance of the capacitor with pure RGO film electrodes is significantly larger than that with the pure GO film, which can be explained by the more efficient charge carrier transfer/storage process due to its enhanced electronic conductivity upon reduction. The capacitor with PPy fibre/RGO film electrodes generally displayed the largest nearly rectangular CV curve, revealing its ideal capacitive behaviour and high capacitance. Its high capacitance is contributed by two key factors including the layered porous structure and the capacitive contribution from the faradaic pseudocapacitance of the polypyrrole fibres. The porous structure of the PPy fibre/RGO film provides larger interface between the electrolyte and the film electrodes, and thus enhances the accessibility for electron storage and transportation. 
The galvanostatic charge/discharge of all three types of supercapacitors at the current density of $1 \mathrm{~A}$ $\mathrm{g}^{-1}$, were tested as showed in Fig.5b. Pure GO and RGO film both showed the near triangle shaped charge/discharge curves (Fig.6a), suggesting that their capacitance are mainly contributed by the electrical double layers (EDLs). It is clear that the capacitor with PPy fibre/RGO film electrodes has the highest discharge capacitance ( $345 \mathrm{~F} \mathrm{~g}^{-1}$ ), which is contributed mainly by the enlarged surface areas and high electric conductivity of the electrodes, as explained above. Such capacitance is comparable to or higher than the reported high performance flexible supercapacitors constructed by various type of flexible electrodes reported previously, including graphene coated cellulose paper $\left(120 \mathrm{~F} \mathrm{~g}^{-1}\right)^{50}$, free standing graphene papers coated by polypyrrole particles via pulse current electrodeposition, $\left(237 \mathrm{~F} \mathrm{~g}^{-1}\right)^{53}$, polyanniline coated carbon nano tubes electrodes $\left(350 \mathrm{~F} \mathrm{~g}^{-1}\right)^{51}$, and graphene/multiwalled CNT paper $\left(265 \mathrm{~F} \mathrm{~g}^{-1}\right)^{54}$ ect. Fig. $5 \mathrm{c}$ reveals the capacitances of all three types of supercapacitors at different discharge current densities. The capacitances of both Pure GO film and RGO film have no significant variation upon the charge/discharge rate due to their EDLs capacitive mechanism, whereas the capacitor composed of PPy fibre/RGO film electrode exhibit slight capacitance decrease at higher charge/discharge rate. This is mainly caused by the incomplete doping/dedoping reaction of PPy fibre, and the pseudocapacitance from the PPy fibre is reduced. Fig. $5 \mathrm{~d}$ shows the Ragone plot of our supercapcitors with PPy/RGO film electrodes and the reported results from other analogical solid state supercapacitors for comparison. The cell with flexible PPy fibre/RGO electrodes displayed considerably high energy density under the medium power density range compared to the reported work. It presented an energy density of $20.6 \mathrm{Wh} \mathrm{kg}^{-1}$ with a power density of $1280 \mathrm{~W} \mathrm{~kg}^{-1}$, which is better than the cell with the PANI fibre/RGO electrodes (986 $\mathrm{W} \mathrm{kg}^{-1}$ and $16.7 \mathrm{Wh} \mathrm{Kg}^{-1}$, ref 52$)^{52}$. 

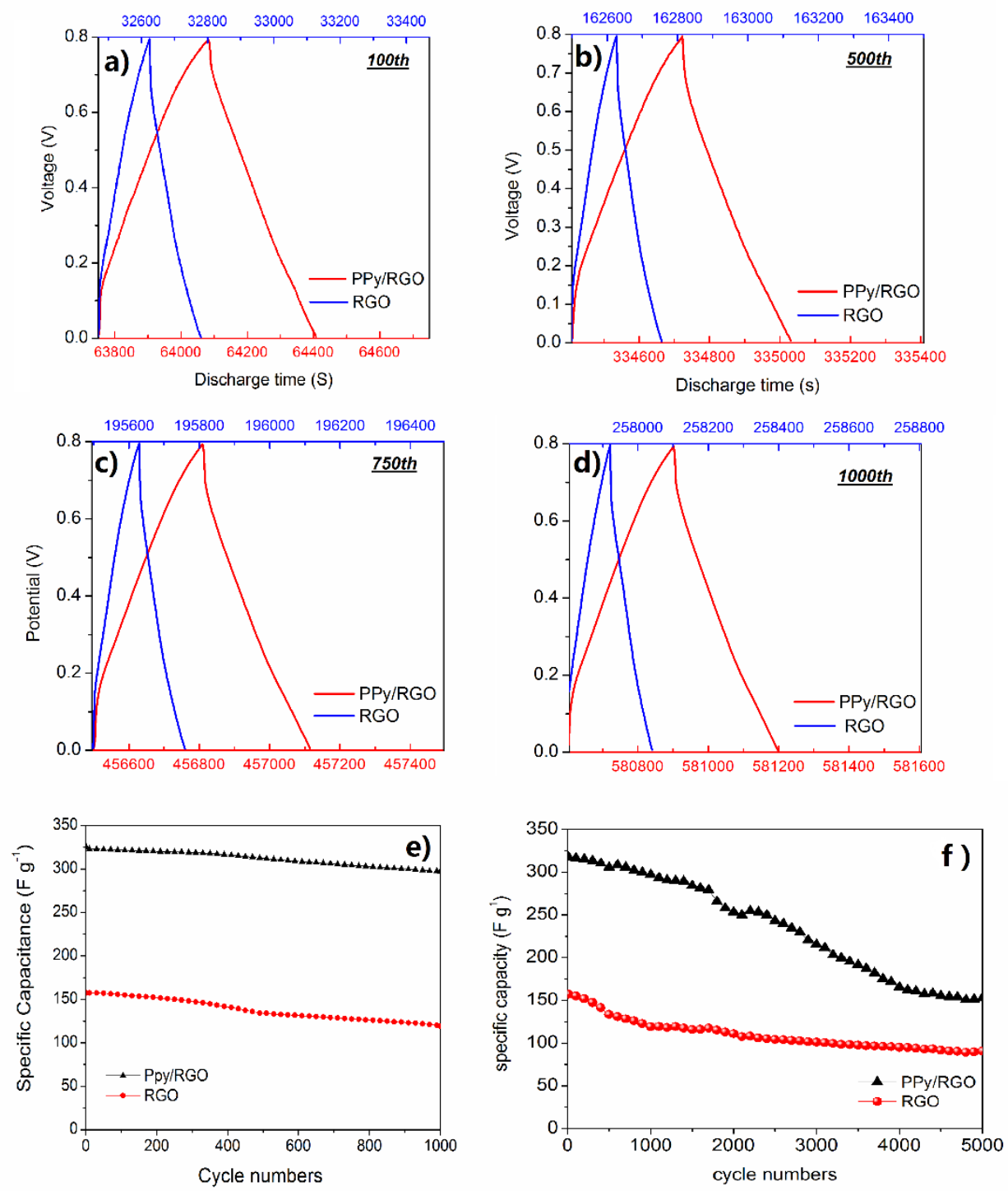

Figure 6. Galvanostatic charge-discharge curves of the symmetric supercapacitors fabricated with PPy/RGO and pure RGO electrodes at $1 \mathrm{~A} \mathrm{~g}^{-1}$ at the different discharge stage (a-d) and the cycling stability (e) 1000 cycles (f) 5000 cycles

The cycling stability of both the supercapacitors composed of PPy fibre/RGO films and pure RGO films are shown in Fig.6. The retention rate of capacitance of both type of supercapacitors are excellent with the first 1000 cycles (Fig.6e). The capacitance retention rate of the capacitor with PPy/RGO film electrodes and pure RGO film electrodes are $91.6 \%$ and $76.8 \%$ after 1000 cycles respectively. It is remarkable that PPy fibre/RGO film electrodes displayed higher capacitance and stable cycling performance when compared with most of the reported supercapacitors composed of chemically or electrochemically synthesised PPy/RGO electrodes ${ }^{20-27}$. The discharge curves at different stages of the cycling process (Fig.6 a-d) revealed that the discharge curve shape of both type 
of capacitors were well retained, indicating the excellent reversibility and stability of their capacitive behaviour. As revealed in the surface morphology of PPy fibre/RGO film (Fig.2d), PPy fibres are sandwiched by the sheer graphene nano sheets layer by layer, which protected PPy fibres from the volumetric swelling and deformation of polymer chains caused by the continuous injection and abjection of counter ions and charge carriers during the cyclic redox reactions ${ }^{55}$. Meanwhile, the protective graphene layers are constituted by the highly conductive $2 \mathrm{D}$ graphene nano sheets with shared edges which effectively helped to improve the facile charge transportation rate of PPy/RGO film electrodes. Therefore, the capacitance drop of such supercapacitor constructed by the multi-layer structured PPy fibre/RGO film electrodes during the initial 1000 cycles is greatly inhibited, and its capacitance is maintained at a relatively high level.

It is also notable that after 1000 cycles the capacitance of the cell with PPy/RGO electrode drops more quickly compared with that of the cell with pure RGO film (Fig.5f). This is mainly due to the decreased redox capacitance contribution from polypyrrole fibres. The prolonged charge/discharge process resulted in the PPy deterioration due to the polymer volume change produced and limited the cycling stability. However, it should be noted that even after 5000 cycles the discharge capacitance of the cell with PPy/RGO film (152 $\left.\mathrm{F} \mathrm{g}^{-1}\right)$ is still higher than the cell with pure RGO film $\left(90 \mathrm{~F} \mathrm{~g}^{-1}\right)$.

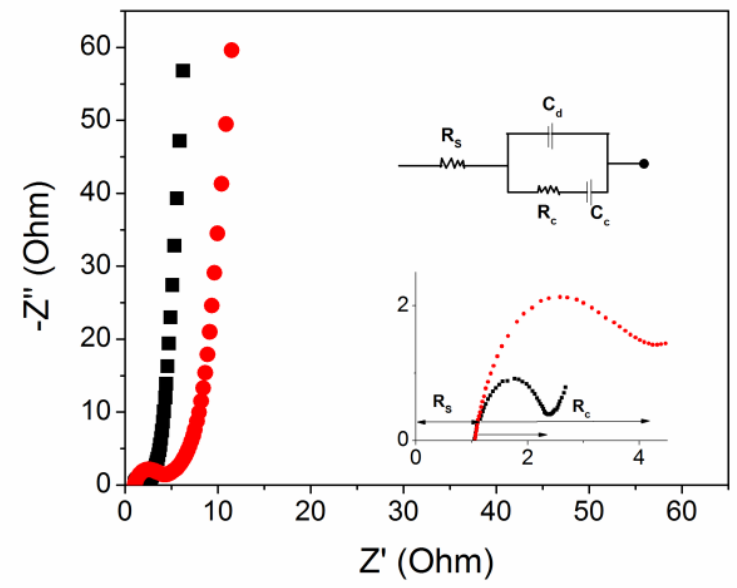

Figure 7. Nyquist plot of flexible supercapacitors constructed by pure RGO film electrode (black square) and PPy fibre/RGO film electrode (Red dot) 
To further investigate the resistance of different supercapacitors, Nyquist plots of electrochemical impedance spectroscopy for both the supercapacitors after 500 cycles are shown in Fig.7. The equivalent circuit of the impedance spectra is presented with several elements, including the resistance generated by the gel electrolyte Rs, the total charge transfer resistance Rc, the EDLs capacitance $\mathrm{Cd}$ and the pseudo-capacitive element $\mathrm{Cp}$. The straight linear parts of the curves at the low frequency regions are related to the ion diffusion process between the two electrodes ${ }^{56}$. The more vertical the curve, the more closely the supercapacitor behaves as an ideal capacitor ${ }^{57}$. According to Fig.7 both of the curves displayed a near vertical line at the low frequency region, suggesting the low ion diffusion resistance inside the capacitor. The arc shaped parts at the high frequency regions generally represent the resistance generated from the charge carrier transportation or namely the charge transfer resistance. It is notable that the pure RGO film is slightly smaller than that of the PPy fibre/RGO film since its charge transfer process occurs purely along the graphene basal plane. This is in correspondence with the electrical conductivity data in Table.1, which showed that the conductivity of pure RGO film is $15.5 \%$ higher than the PPy/RGO film. However, the additional PPy fibres state the advantages about the addition of PPy fibre.

\section{Conclusion}

Multi-layered flexible polypyrrole fibres/reduced graphene oxide films were prepared by a simple vacuum filtration method followed by HI reduction, which featured with excellent mechanical properties, high conductivity and excellent capacitive performances. The restack of graphene nano sheets were significantly mitigated due to the evenly distributed polypyrrole nano fibres in between graphene nano sheets. The supercapacitors constructed by the $\mathrm{PPy} / \mathrm{RGO}$ film with the gel $\mathrm{PVA} / \mathrm{H}_{3} \mathrm{PO}_{4}$ electrolyte performed much better than the pure RGO film and GO film with a galvanostatic discharge capacitance up to $345 \mathrm{~F} \mathrm{~g}^{-1}$ at a current density of $1 \mathrm{~A} \mathrm{~g}^{-1}$. The enhanced capacitance can be ascribed to two contributing factors. Firstly, the polypyrrole fibre brings in the pseudo-capacitance from its reversible redox reaction. Second, the polypyrrole fibre helps to create the porous structures between graphene layers and thus enlarge accessible area of the electrodes. The supercapacitor constructed 
using the PPy/RGO film electrodes also displayed very stable cycling performance with only $9.4 \%$ capacitance drop after 1000 cycles. The long term cycling stability is also tested for up to 5000 cycles, the supercapacitor with PPy/RGO film electrodes presented significantly higher capacitance throughout the discharge process. This can be explained by the excellent charge transfer accessibility of the graphene nano sheets and its protective effects on the polypyrrole fibres.

\section{References}

1. D. Wei, S. J. Wakeham, T. W. Ng, M. J. Thwaites, H. Brown and P. Beecher, Electrochemistry Communications, 2009, 11, 2285-2287.

2. B. Scrosati, The Chemical Record, 2005, 5, 286-297.

3. G. Pistoia, Battery operated devices and systems: From portable electronics to industrial products, Elsevier, 2008.

4. Y. G. Guo, J. S. Hu and L. J. Wan, Advanced Materials, 2008, 20, 2878-2887.

5. L. Kou, T. Huang, B. Zheng, Y. Han, X. Zhao, K. Gopalsamy, H. Sun and C. Gao, Nature communications, 2014, 5.

6. M. Winter and R. J. Brodd, Chemical reviews, 2004, 104, 4245-4270.

7. B. E. Conway, 1999.

8. L. L. Zhang, R. Zhou and X. Zhao, Journal of Materials Chemistry, 2010, 20, 5983-5992.

9. A. K. Geim and K. S. Novoselov, Nature materials, 2007, 6, 183-191.

10. M. Pumera, Energy \& Environmental Science, 2011, 4, 668-674.

11. T. Huang, B. Zheng, L. Kou, K. Gopalsamy, Z. Xu, C. Gao, Y. Meng and Z. Wei, RSC Advances, 2013, 3, 23957-23962.

12. C. Liu, Z. Yu, D. Neff, A. Zhamu and B. Z. Jang, Nano letters, 2010, 10, 4863-4868.

13. H. Chen, M. B. Müller, K. J. Gilmore, G. G. Wallace and D. Li, Advanced Materials, 2008, 20, 3557-3561.

14. X. Yang, C. Cheng, Y. Wang, L. Qiu and D. Li, Science, 2013, 341, 534-537.

15. X. Yan, Z. Tai, J. Chen and Q. Xue, Nanoscale, 2011, 3, 212-216.

16. Z. Tai, X. Yan, J. Lang and Q. Xue, Journal of power sources, 2012, 199, 373-378.

17. T.-K. Hong, D. W. Lee, H. J. Choi, H. S. Shin and B.-S. Kim, Acs Nano, 2010, 4, 3861-3868.

18. J.-Z. Wang, C. Zhong, S.-L. Chou and H.-K. Liu, Electrochemistry Communications, 2010, 12, 1467-1470.

19. D. Yu, K. Goh, H. Wang, L. Wei, W. Jiang, Q. Zhang, L. Dai and Y. Chen, Nature nanotechnology, 2014.

20. X. Yang, J. Zhu, L. Qiu and D. Li, Advanced Materials, 2011, 23, 2833-2838.

21. D. Yu and L. Dai, The Journal of Physical Chemistry Letters, 2009, 1, 467-470.

22. G. A. Snook, P. Kao and A. S. Best, Journal of power sources, 2011, 196, 1-12.

23. X. Zhao, B. M. Sánchez, P. J. Dobson and P. S. Grant, Nanoscale, 2011, 3, 839-855.

24. A. G. MacDiarmid, Synthetic Metals, 1997, 84, 27-34.

25. S. Biswas and L. T. Drzal, Chemistry of Materials, 2010, 22, 5667-5671.

26. L. L. Zhang, S. Zhao, X. N. Tian and X. Zhao, Langmuir, 2010, 26, 17624-17628.

27. Y. Xu, Z. Lin, X. Huang, Y. Liu, Y. Huang and X. Duan, ACS nano, 2013, 7, 4042-4049.

28. D. Zhang, X. Zhang, Y. Chen, P. Yu, C. Wang and Y. Ma, Journal of Power Sources, 2011, 196, 5990-5996.

29. C. Zhu, J. Zhai, D. Wen and S. Dong, Journal of Materials Chemistry, 2012, 22, 6300-6306. 
30. H.-H. Chang, C.-K. Chang, Y.-C. Tsai and C.-S. Liao, Carbon, 2012, 50, 2331-2336.

31. C. Xu, J. Sun and L. Gao, Journal of Materials Chemistry, 2011, 21, 11253-11258.

32. L. L. Zhang, X. Zhao, M. D. Stoller, Y. Zhu, H. Ji, S. Murali, Y. Wu, S. Perales, B. Clevenger and R. S. Ruoff, Nano letters, 2012, 12, 1806-1812.

33. Y. Xu, H. Bai, G. Lu, C. Li and G. Shi, Journal of the American Chemical Society, 2008, 130, 5856-5857.

34. C. Zhao, C. Wang, Z. Yue, K. Shu and G. G. Wallace, ACS applied materials \& interfaces, 2013, 5, 9008-9014.

35. S. Stankovich, D. A. Dikin, R. D. Piner, K. A. Kohlhaas, A. Kleinhammes, Y. Jia, Y. Wu, S. T. Nguyen and R. S. Ruoff, Carbon, 2007, 45, 1558-1565.

36. X. Zhang, J. Zhang, W. Song and Z. Liu, The Journal of Physical Chemistry B, 2006, 110, 11581165.

37. Q. Wu, Y. Sun, H. Bai and G. Shi, Physical Chemistry Chemical Physics, 2011, 13, 11193-11198.

38. D. A. Dikin, S. Stankovich, E. J. Zimney, R. D. Piner, G. H. Dommett, G. Evmenenko, S. T. Nguyen and R. S. Ruoff, Nature, 2007, 448, 457-460.

39. S. Pei, J. Zhao, J. Du, W. Ren and H.-M. Cheng, Carbon, 2010, 48, 4466-4474.

40. C. Gómez-Navarro, R. T. Weitz, A. M. Bittner, M. Scolari, A. Mews, M. Burghard and K. Kern, Nano letters, 2007, 7, 3499-3503.

41. J. Zhang, H. Yang, G. Shen, P. Cheng, J. Zhang and S. Guo, Chemical Communications, 2010, 46, 1112-1114.

42. O. C. Compton and S. T. Nguyen, Small, 2010, 6, 711-723.

43. D. Yang, A. Velamakanni, G. Bozoklu, S. Park, M. Stoller, R. D. Piner, S. Stankovich, I. Jung, D. A. Field and C. A. Ventrice Jr, Carbon, 2009, 47, 145-152.

44. S. Yumitori, Journal of materials science, 2000, 35, 139-146.

45. S. Bose, T. Kuila, M. E. Uddin, N. H. Kim, A. K. Lau and J. H. Lee, Polymer, 2010, 51, 5921-5928.

46. Y.-C. Liu, B.-J. Hwang, W.-J. Jian and R. Santhanam, Thin Solid Films, 2000, 374, 85-91.

47. A. Ferrari, J. Meyer, V. Scardaci, C. Casiraghi, M. Lazzeri, F. Mauri, S. Piscanec, D. Jiang, K. Novoselov and S. Roth, Physical review letters, 2006, 97, 187401.

48. L. Malard, M. Pimenta, G. Dresselhaus and M. Dresselhaus, Physics Reports, 2009, 473, 51-87.

49. Y. J. Kang, H. Chung, C.-H. Han and W. Kim, Nanotechnology, 2012, 23, 065401.

50. Z. Weng, Y. Su, D. W. Wang, F. Li, J. Du and H. M. Cheng, Advanced Energy Materials, 2011, 1, 917-922.

51. C. Meng, C. Liu, L. Chen, C. Hu and S. Fan, Nano letters, 2010, 10, 4025-4031.

52. Q. Wu, Y. Xu, Z. Yao, A. Liu and G. Shi, Acs Nano, 2010, 4, 1963-1970.

53. A. Davies, P. Audette, B. Farrow, F. Hassan, Z. Chen, J.-Y. Choi and A. Yu, The Journal of Physical Chemistry C, 2011, 115, 17612-17620.

54. X. Lu, H. Dou, B. Gao, C. Yuan, S. Yang, L. Hao, L. Shen and X. Zhang, Electrochimica Acta, 2011, 56, 5115-5121.

55. T. Otero and J. Padilla, Journal of Electroanalytical Chemistry, 2004, 561, 167-171.

56. R. Kötz, M. Hahn and R. Gallay, Journal of Power Sources, 2006, 154, 550-555.

57. W. Sugimoto, H. Iwata, K. Yokoshima, Y. Murakami and Y. Takasu, The Journal of Physical Chemistry B, 2005, 109, 7330-7338. 